\title{
Vigilancia tecnológica del sector agroindustrial
}

\author{
Juan Manuel Andrade \\ Corporación Universitaria Minuto de Dios, Colombia \\ jandrandenav@uniminuto.edu.co \\ Elías Ramírez Plazas \\ Universidad Surcolombiana, Colombia \\ elias.ramirez@usco.edu.co \\ Alexander Quintero \\ Universidad Surcolombiana, Colombia \\ alequin@usco.edu.co
}

\section{Resumen}

La gestión científica y tecnológica en la actualidad es indispensable para el desarrollo regional y nacional, especialmente en sectores con importancia y proyección tanto social como económica. Por tal motivo, el presente artículo presenta un estudio de Vigilancia Tecnológica para el sector agroindustrial a partir de ecuaciones de búsqueda en la base de datos Scopus. Asimismo, se definió un plan para el estudio que contempla cuatro etapas: producción científica, autores destacados en la producción científica, países con mayor número de publicaciones e instituciones destacadas con producción científica. Finalmente, se evidenció la tendencia creciente durante la década estudiada en investigaciones relacionadas con la utilidad de subproductos y propiedades de los productos de origen agropecuario.

Palabras clave: Ecuación de búsqueda, agroindustria, vigilancia tecnológica, producción científica.

\section{Technological surveillance of the agro-industrial sector}

\begin{abstract}
Scientific and technological management are currently imperative for regional and national development, especially in sectors of social and economic importance. For this reason, the present article presents a Technological Watch study for the agroindustrial sector, based on search equations in the Scopus database. Also, the plan for the study was defined as four stages: scientific production, outstanding authors in scientific production, countries with the highest number of publications and institutions with scientific production. Finally, the growing trend during the decade studied was evidenced in research related to the utility of byproducts and properties of products of agricultural origin.
\end{abstract}

Keywords: Equation Research, agroindustry, Technology Watch, Scientific production.

\section{Introducción}

Las dinámicas del comercio internacional han sido relevantes en la determinación de las estructuras productivas de sectores económicos en la gran mayoría de los países, ocasionando que los distintos sectores productivos se replanteen constantemente, especialmente cuando existe un número importante de medianas y pequeñas empresas que necesitan avanzar tecnológicamente, a 
un ritmo competitivo y desarrollar competencias dinámicas (Rivera, 2015). Al respecto, recientes experiencias sugieren que una manera exitosa de reaccionar a este entorno consiste en incrementar las inversiones en I+D+i para la mejora de productos y procesos (Sandino-Vargas, 2014).

En esa lógica, en el auge de las nuevas tecnologías, tendencias como la vigilancia tecnológica se han venido imponiendo como estrategias de competitividad, en gran medida, justificadas por el volumen de información relevante necesaria para la toma de decisiones (Palop \& Vicente, 1999; Ramírez-Calvo et al., 2013).

Reconociendo el valor potencial del análisis bibliométrico (Daim et al., 2006; Lee, Kim \& Shin, 2017), muchos autores han centrado sus estudios en herramientas como la minería de datos (Lee, Kim, Song, Park \& Shin, 2014) y el análisis de patentes (Lee, Yoon \& Park, 2009).

La vigilancia tecnológica (en adelante, VT) puede definirse como el proceso sistemático y organizado de búsqueda, captación y análisis de información de carácter tecnológico, comercial, competitivo y normativo a nivel nacional e internacional, que permite anticiparse para esclarecer las acciones a través de las decisiones, pasando antes por la apropiación y llegando a un aprendizaje colectivo o de la organización (Cinertya Consulting, 2010; Du Toit, 2013; Strategic and Competitive Intelligence Professionals, 2014).

La aplicación de este modelo metodológico tiene su fundamentación y origen en los modelos prospectivos, que a través de su aplicación permite la identificación de factores de cambio o factores portadores de futuro, que tendrán un impacto directo en el proceso de planeación estratégica y que definirá el escenario deseado para una empresa o territorio.

LaVTnacedelanecesidad delasorganizaciones de observar su entorno y tener la capacidad de responder a los cambios que se producen, y que generalmente las afectan. En otras palabras, es el esfuerzo sistemático y organizado por la empresa de observación, captación, análisis, difusión precisa y recuperación de información sobre los hechos del entorno económico, tecnológico, social o comercial, relevantes para la misma por poder implicar una oportunidad u amenaza, con objeto de poder tomar decisiones con menor riesgo y poder anticiparse a los cambios (Escorsa \& Maspons, 2001; Du Toit, 2013; Villarroel, 2015).

A pesar de que existen diferentes tipologías de vigilancia tecnológica (ver figura 1), en el presente estudio se enfoca en la referente a los avances en producción científica y tecnológica.

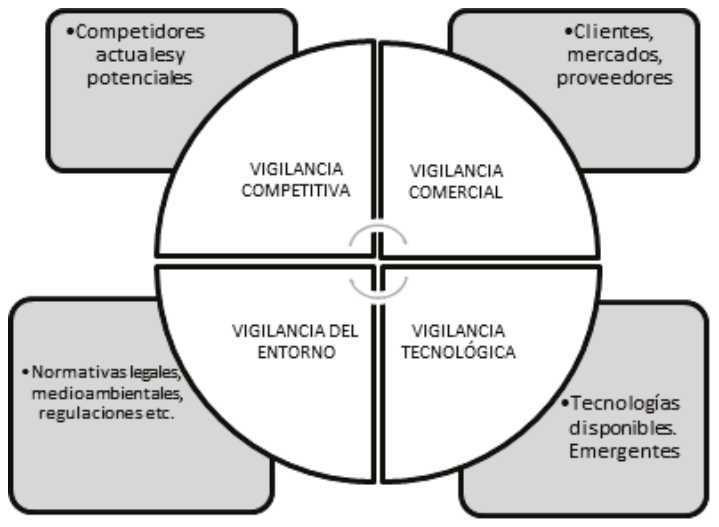

Figura 1. Tipología de la Vigilancia tecnológica Fuente: Elaboración propia

Lo anterior dado que VT está centrada en los avances del estado de la técnica y de la tecnología de disponible y emergente en cualquier sector empresarial. Esta permite identificar tecnologías en declive y garantizar los procesos de transferencia tecnológica, de igual forma en el área de investigación y desarrollo permite establecer el nivel inventivo y de patentabilidad de una innovación (Cinertya Consulting, 2010; Strategic and Competitive Intelligence Professionals, 2014).

Así, el sector puede organizar la vigilancia en torno a la información sobre los competidores actuales y/o potenciales, y de aquellos productos sustitutos, realizando un análisis y seguimiento del destino de sus inversiones, sus productos, circuitos de distribución, tiempos de respuesta, tipo de clientes y grado de satisfacción, su organización y capacidad financiera. La cadena de valor del sector y su participación en dicha cadena de valor (Porter \& Cunningham, 2005). En ese entendido, se torna relevante conocer 
las tendencias científicas relacionadas con la agroindustria, teniendo en cuenta que se constituye en una apuesta productiva nacional de la que depende ampliamente la economía colombiana, $y$ que en años recientes ha realizado ingentes esfuerzos por implementar desarrollos de base tecnológica que le permitan reorientar su cadena productiva hacia productos con valor agregado.

Por lo tanto, aspectos como la producción científica, autores destacados en la producción científica, países con mayor número de publicaciones e instituciones destacadas con producción científica, serán objeto de estudio en este artículo.

\section{Materiales y métodos}

Para la realización de estudios de vigilancia tecnológica existen varios procedimientos descritos por autores reconocidos, la metodología usada para el desarrollo del estudio se expone a continuación (Berges-García, Meneses-Chaus \& Martínez-Ortega, 2016; Giménez-Toledo, \& Román-Román, 2001).

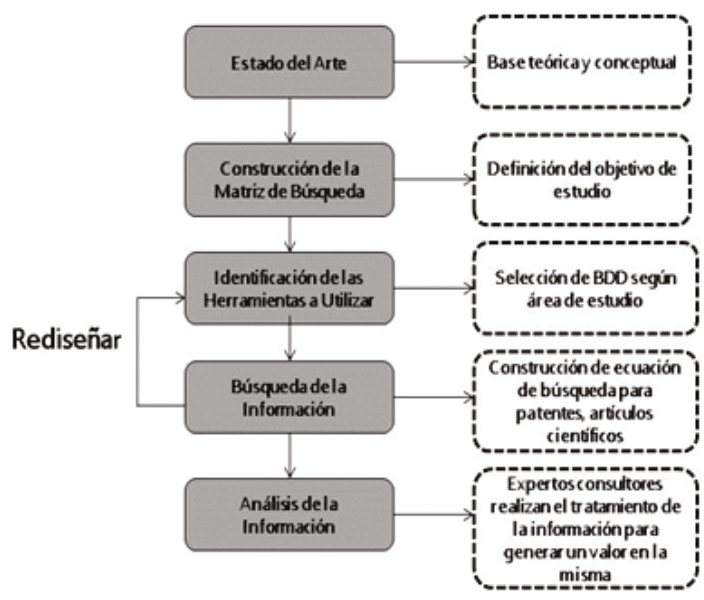

Figura 2. Metodología General Para Vigilancia Tecnológica

Fuente: Elaboración propia

\section{Construcción de la matriz de búsqueda}

La construcción de la matriz de búsqueda está orientada a la definición del alcance del estudio. Este se desarrolla en conjunto entre expertos e investigadores, que por su conocimiento y experiencia aportan elementos clave a tener en cuenta en el desarrollo del mismo.
La matriz se representa a través de un mapa conceptual que permite a los vigías y analistas orientar sus esfuerzos en búsqueda del objetivo planteado (ver figura 3).

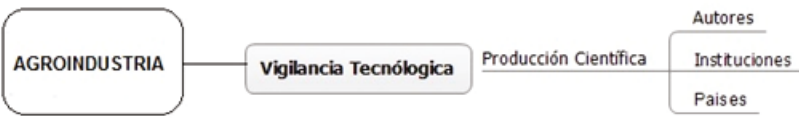

Figura 3. Matriz de búsqueda para los cuatro frutos promisorios

Fuente: Elaboración propia

\section{Identificación de las herramientas a utilizar}

Para la elaboración del artículo se identificó y accedió a las principales bases de datos que alberga información relacionada con el tema de investigación, utilizando:

\section{- A nivel científico}

Scopus: La mayor base de datos de citas y resúmenes de literatura revisada por pares: revistas científicas, libros y actas de congresos. Cuenta con herramientas inteligentes para rastrear, analizar y visualizar la investigación, ofreciendo una visión general de la producción mundial de investigación en los campos de la ciencia, la tecnología, la medicina, las ciencias sociales y las artes y las humanidades.

\section{Búsqueda de la información}

Teniendo en cuenta que es importante establecer dentro de las búsquedas los parámetros que se convertirán en indicadores, y de este modo lograr una homogeneidad en la información. Para la exploración de la información, se establecieron diferentes ecuaciones lógicas basadas en tesauros y operadores booleanos, con el fin de obtener la mayor cantidad de datos científicos, tecnológicos, comerciales y conceptuales validados.

\section{Identificación y definición de tesauros}

Inicialmente se construye un tesauro o listado de palabras con significados similares o sinónimos en inglés, los cuales permitirán establecer relaciones directas o indirectas con los conceptos a consultar, y a su vez, construir de forma más precisa las ecuaciones de búsqueda. 
Para tal propósito se utilizaron herramientas validadas internacionalmente, que agrupan los tesauros a nivel mundial, como el SCIMago Journal \& Country Ranky el Map Generator, que muestra de manera gráfica la interacción que presentan las diferentes áreas del conocimiento por país y años, permitiendo generar con mayor facilidad las predicciones de búsqueda dado que está integrado con Scopus.

\section{Construcción de las ecuaciones mediante el uso de operadores}

En la construcción de las ecuaciones de búsqueda se tuvo en cuenta tanto los tesauros, como los operadores boléanos, estimando una construcción de tres ecuaciones para búsqueda de artículos científicos y otras tres para búsqueda de patentes. Los operadores permitieron enfocar la exploración, vinculando términos de búsqueda y definiendo la relación entre ellos.

Construcción de los indicadores para el análisis bibliométrico

La construcción de los indicadores está fundamentada en un análisis bibliométrico, que engloba el estudio de los aspectos cuantitativos de la producción, diseminación y uso de la información que se tiene registrada en bases de datos especializadas, tratando de identificar:

* La frecuencia de uso de las palabras y frases, tanto en textos y artículos científicos, como en patentes, teniendo en cuenta la distribución por autores, por tipo de documento, por instituciones, y por países.

* Las características de la productividad autoral, medida por el número de documentos publicados o por el grado de colaboración.

* Las características de las fuentes publicadas, incluyendo la distribución de los documentos por disciplinas

La construcción para cada bloque será la siguiente:

o Bloque de producción bibliográfica

o Publicaciones en el tiempo

o Palabras claves

o Instituciones con mayor número de publicaciones o Países con mayor número de publicaciones

La ecuación de busqueda utilizada fue ( ( ( TITLE-ABS-KEY (agroindustria ) ) OR ( TITLEABS-KEY ( agro-industria ) ) OR ( TITLE-ABSKEY ( agroindustry ) ) OR ( TITLE-ABS-KEY ( agro-industrial ) ) OR ( TITLE-ABS-KEY ( agroindustry ) ) OR ( TITLE-ABS-KEY ( agribusiness )) OR ( TITLE-ABS-KEY ( agri-food industry )) OR ( TITLE-ABS-KEY ( agroaliment* $)$ )) OR ( TITLE-ABS-KEY ( "agri-food sector" ) ) OR ( TITLE-ABS-KEY ( agro-food ) ) ) AND PUBYEAR > 2004

\section{Análisis de la información}

Para el procesamiento de la información se utilizó el software de vigilancia tecnológica Matheo Analizer, mediante el cual se pudo obtener, una manipulación precisa de los archivos planos exportados de cada una de las bases de datos, el software maneja una interfaz que permite una mejor visualización y manipulación de los datos obtenidos generando relaciones de intercambio para grupos de datos.

Luego de obtener la información según los indicadores construidos, se analizaron los principales avances científicos publicados en artículos de revistas indexadas, con el fin de:

o Buscar los países líderes o más relevantes en el desarrollo de tecnologías parecidas o similares a la cobijada en la invención o en donde parece haber una actividad industrial o comercial en relación con la misma.

o Países donde tecnologías similares o parecidas están emergiendo (en proceso de consolidación industrial y/o comercial).

o Investigar sobre las solicitantes actuales de patentes más relevantes (empresas, universidades, centros de investigación y afines) en el desarrollo de tecnologías similares.

\section{Resultados y discusión}

\section{Producción científica}

Los resultados obtenidos a través de la ecuación de búsqueda desarrollada muestran para el periodo 2005 - 2016 un total de 7.858 
productos de conocimiento relacionados con el área de estudio y producción científica con una tendencia creciente para el sector agroindustrial (ver figura 4).

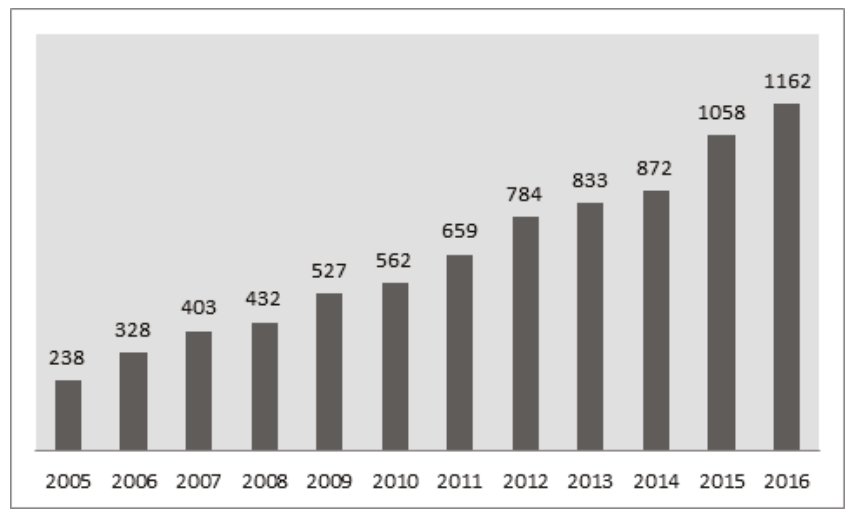

Figura 4. Producción científica para sector agroindustrial (2005 - 2015)

Fuente: Elaboración propia

Entrelos productos de conocimiento secuentan 6.819 artículos científicos, 717 documentos derivados de conferencias y 322 capítulos de libros. Las áreas de conocimiento relacionadas comprenden la agricultura y ciencias biológicas con 3.873 documentos, ciencias sociales con 1.799, ciencias ambientales 1.426, gestión de negocios y contabilidad, bioquímica y genética molecular con 760 .

A partir de estas áreas temáticas, se obtienen las siguientes tendencias de investigación que marcan la evolución del sector (ver figura 5).

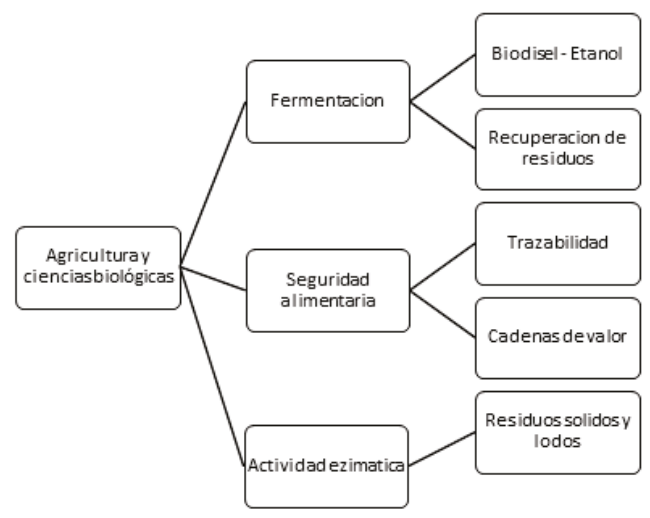

Figura 5. Tendencias de investigación para agricultura y ciencias biológicas

Fuente: Elaboración propia
Para el campo de la agricultura y ciencias biológicas, las tres principales subáreas de investigación son fermentación, seguridad alimentaria y actividad enzimática. En la primera se destaca la producción de biodiesel, etanol o bioetanol a partir de lignocelulosa derivada de plantas procesadoras de caña panelera $u$ otros cultivos. Su principal foco de investigación es el desarrollo de equipos de bajo costo, pues la limitación que tiene el procesamiento de lignocelulosa es la ausencia de tecnológica accesible a medianos y pequeños productores. Otro aspecto es la recuperación de residuos basado en fermentación enzimática, ultrasonido con Aspergillus japonicus PJ01 y Bacillus subtilis NX-2.

Frente a la seguridad alimentaria, la investigación en el área de trazabilidad se basa en soluciones TIC para el sector agroindustrial, con más cantidad y calidad de información, mejorando las expectativas del consumidor en cuanto a seguridad alimentaria y calidad del producto. Para el caso de la subárea de cadenas de valor la investigaciones se centran en la satisfacción del consumidor final y su percepción de valor en el producto agroindustrial que consume, de igual forma el enfoque se centra en el estudio de la producción a pequeña escala y la fragmentación de mercados que rompe las cadenas de valor.

En cuanto a la subárea de actividad enzimática, se desataca la recuperación de residuos sólidos y lodos a través de enzimas hidrolíticas, que permiten la remoción de grasas y aceites para la recuperación por medio de otros tratamientos posteriores de bajo costo aplicados a residuos agroindustriales.

Para el área ciencias ambientales se destacan dos subáreas de investigación: biomasa, sostenibilidad y gestión de residuos líquidos (ver figura 6). En cuanto a la biomasa, la subárea de biogás se enfoca en digestión anaeróbica con aplicación de algas a través de la evaluación del potencial bioquímico de metano para el incremento de su rendimiento. 
En cuanto a los bioplásticos, el foco de investigación es la producción de Polihidroxialcanoato "bioplástico". Este polímero presenta la propiedad de degradabilidad, basado en el tratamiento de desechos de la industria de papel y la acidificación a través de bacterias en diferentes etapas de los desechos. La biodegradación se orienta a la modelación cinética de biodigestión de residuos, y la biodegradación por microorganismos como Burkholderia cepacia y organismos halófilos.

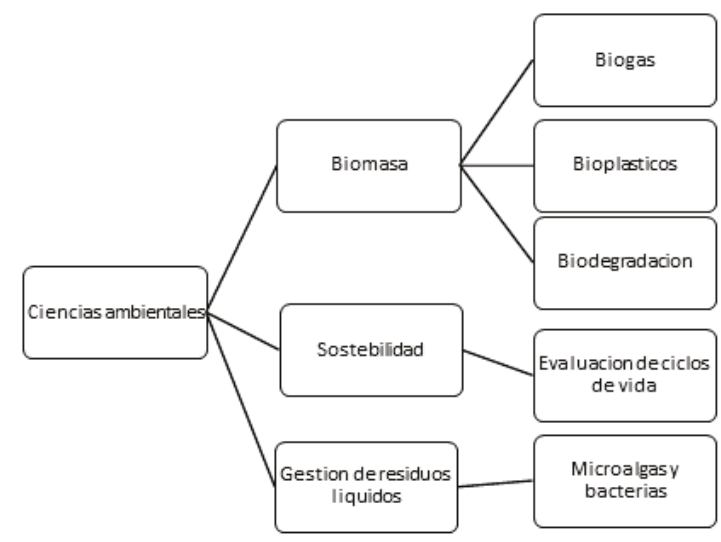

Figura 6. Tendencias de investigación para el área temática ciencias ambientales

Fuente: Elaboración propia

Para la subárea de sostenibilidad, la evaluación de ciclos de vida se orienta en la evaluación de cadenas de valor y proveeduría de productos agroindustriales para la identificación de puntos críticos de impacto ambiental que deban ser intervenidos con el fin de mejorar la productividad y sostenibilidad de los procesos productivos.

Por último en la subárea de gestión de residuos líquidos, sobresale la investigación de microalgas y bacterias para la producción de biocombustibles a través de agregación de biomasa de microalgas y baterías Chlorella sorokiniana. El tratamiento combinado facilita la eliminación de carga lipídica de residuos líquidos.

Se estima que la producción de algas es diez veces más alta que las plantas cultivadas en tierras, y presenta independencia de tierra para cultivo. La biomasa de algas es rica en lípidos, proteínas y almidón, que pueden ser convertidos en energía usando procesos térmicos, bioquímicos y esterificación de ácidos grasos para producir biodiesel, incluso pude servir para la producción de biocombustibles de segunda generación como el metano.

En cuanto al área temática de bioquímica y genética molecular (ver figura 7), se destacan dos subáreas de investigación; la farmacéutica y la síntesis enzimática. Para la subárea farmacéutica, la investigación se enfoca en la identificación y caracterización de principios activos de materias primas agrícolas $y$ residuos agroindustrial para la producción de productos cosméticos, entre los principios activos se destacan los fenoles y carotenoides principalmente de café y polifenoles antioxidantes de pericarpio de palta (aguacate) entre otros.

La investigación en formulación médica se orienta en la obtención de ácidos fenólicos tales como ácido cafeico, ácido p-cumárico y ácido ferúlico por diferentes vías. Los ensayos se han realizado mediante eruloil esterasa derivada de aspergillus niger. Los desechos utilizados para la obtención de estos principios con potencial farmacéutico fueron pulpa de café, pulpa de manzana y paja de trigo.

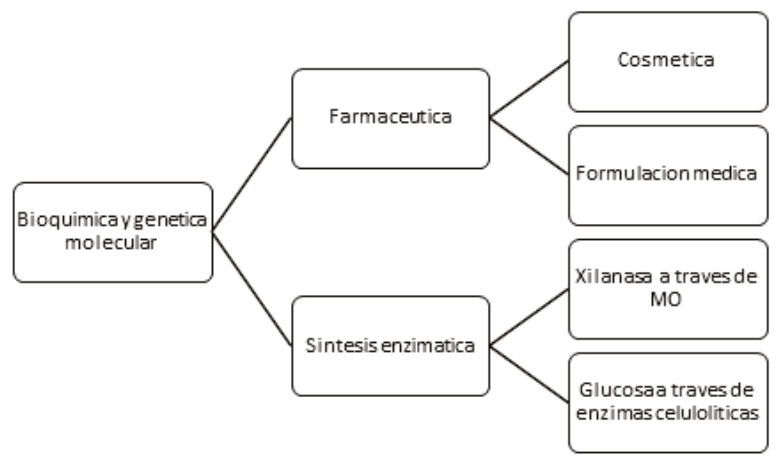

Figura 7. Tendencias de investigación para bioquímica y genética molecular

Fuente: Elaboración propia

Para el subárea de síntesis enzimática, existe especial interés en la obtención de xilanasa a partir de desechos agroindustriales, mediante microrganismos como aspergillus niger, aspergillus orizae, aspergillus japónico, penicillium canescens, talaromices y bacilos circulans. Las xilanasas pertenecen a los 
pentosanasas, un grupo de enzimas que descomponen componentes de la matriz de la pared celular de las plantas, y se utiliza en panadería principalmente como una enzima de hornear para mejorar cualidades de masa (trabajabilidad, estabilidad) y para optimizar el producto (estabilización de la corteza y el volumen).

\section{Autores destacados en la producción científica}

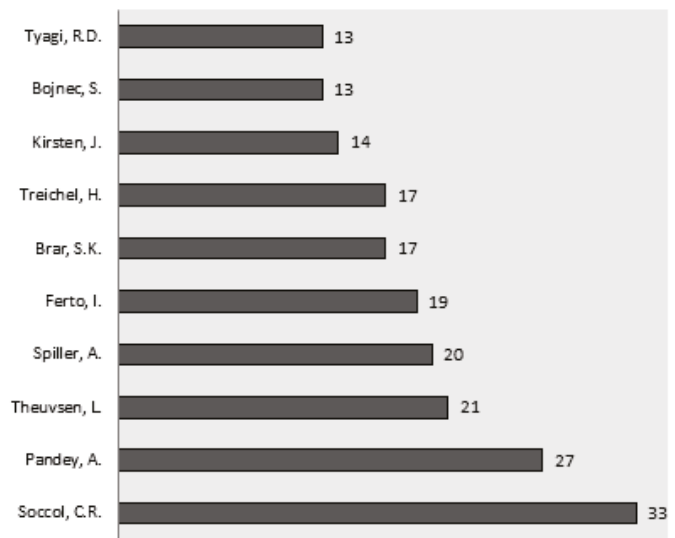

Figura 8. Autores destacados en el sector agroindustrial (2005 - 2015)

Fuente: Elaboración propia

La producción científica relacionada con el sector agroindustrial (ver figura 8) está liderada por el científico Carlos Ricardo Soccol, de la Universidad Federal de Paraná, con 33 publicaciones relacionadas con agroindustria, su área de investigación principal es la bioquímica y la genética molecular (Coral Medina et al., 2015; Da Luz Costa et al., 2015; De Melo et al., 2015; Medina-Macedo et al., 2015; Moreira et al., 2015; Liguori et al., 2015) (ver tabla 1).
Tabla 1. Producción científica reciente C. R. Soccol

\begin{tabular}{|c|c|c|}
\hline Artículo & Año & Revista \\
\hline $\begin{array}{l}\text { High levels of genetic diversity } \\
\text { through pollen flow of the } \\
\text { coniferous Araucaria angustifolia: } \\
\text { a landscape level study in } \\
\text { Southern Brazil }\end{array}$ & 2015 & $\begin{array}{c}\text { Tree Genetics and } \\
\text { Genomes }\end{array}$ \\
\hline $\begin{array}{l}\text { Lignin preparation from oil palm } \\
\text { empty fruit bunches by } \\
\text { sequential acid/alkaline } \\
\text { treatment - A biorefinery } \\
\text { approach }\end{array}$ & 2015 & Bioresource Technology \\
\hline $\begin{array}{l}\text { Conducting starter culture- } \\
\text { controlled fermentations of coffee } \\
\text { beans during on-farm wet } \\
\text { processing: Growth, metabolic } \\
\text { analyses and sensorial effects }\end{array}$ & 2015 & $\begin{array}{l}\text { Food Research } \\
\text { International }\end{array}$ \\
\hline $\begin{array}{l}\text { Novel spectrophotometric } \\
\text { method for detection and } \\
\text { estimation of butanol in acetone- } \\
\text { butanol-ethanol fermenter }\end{array}$ & 2015 & Talanta \\
\hline $\begin{array}{l}\text { Callus Growth Kinetics of Physic } \\
\text { Nut (Jatropha curcas L.) and } \\
\text { Content of Fatty Acids from } \\
\text { Crude Oil Obtained In Vitro }\end{array}$ & 2015 & $\begin{array}{l}\text { Applied Biochemistry } \\
\text { and Biotechnology }\end{array}$ \\
\hline $\begin{array}{l}\text { Dynamics of ethanol production } \\
\text { from deproteinized whey by } \\
\text { Kluyveromyces marxianus: An } \\
\text { analysis about buffering capacity, } \\
\text { thermal and nitrogen tolerance }\end{array}$ & 2015 & $\begin{array}{l}\text { Brazilian Archives of } \\
\text { Biology and } \\
\text { Technology }\end{array}$ \\
\hline $\begin{array}{c}\text { Second generation ethanol } \\
\text { production from brewers' spent } \\
\text { grain }\end{array}$ & 2015 & Energies \\
\hline
\end{tabular}

Fuente: Elaboración propia

El segundo autor con mayor cantidad de publicaciones es Ashok Pandey, de la división de biotecnología del instituto Nacional Interdisciplinario de Ciencia y Tecnología, en Thiruvananthapuram, India. Coautor de Soccol en algunas publicaciones. Sus investigaciones se han centrado en la evaluación de métodos de fermentación y obtención de antioxidantes (Khare, Pandey \& Larroche, 2015; Prabisha et al., 2015; Sajitha et al., 2015; Sajna et al., 2015; Sindhu et al., 2015; Varsha et al., 2015; Vivek, Pandey \& Binod, 2015) (ver tabla 2). 
Tabla 2. Producción científica reciente A. Pandey

\begin{tabular}{|c|c|c|}
\hline Artículo & Año & Revista \\
\hline $\begin{array}{l}\text { Biological valorization of pure } \\
\text { and crude glycerol into 1,3- } \\
\text { propanediol using a novel } \\
\text { isolate Lactobacillus brevis } \\
\text { N1E9.3.3 }\end{array}$ & 2015 & Bioresource Technology \\
\hline $\begin{array}{l}\text { A novel crude glycerol assisted } \\
\text { surfactant pretreatment strategy } \\
\text { of chili post-harvest residue for } \\
\text { bioethanol production }\end{array}$ & 2015 & Biofuels \\
\hline $\begin{array}{l}\text { Crude oil biodegradation aided } \\
\text { by biosurfactants from } \\
\text { Pseudozyma sp. NII } 08165 \text { or its } \\
\text { culture broth }\end{array}$ & 2015 & Bioresource Technology \\
\hline $\begin{array}{l}\text { 2,4-Di-tert-butyl phenol as the } \\
\text { antifungal, antioxidant } \\
\text { bioactive purified from a newly } \\
\text { isolated Lactococcus sp. }\end{array}$ & 2015 & $\begin{array}{c}\text { International Journal of } \\
\text { Food Microbiology }\end{array}$ \\
\hline $\begin{array}{l}\text { Production and characterization } \\
\text { of PHB from a novel isolate } \\
\text { Comamonas sp. from a dairy } \\
\text { effluent sample and its } \\
\text { application in cell culture }\end{array}$ & 2015 & $\begin{array}{c}\text { Biochemical } \\
\text { Engineering Journal }\end{array}$ \\
\hline $\begin{array}{l}\text { Current perspectives in } \\
\text { enzymatic saccharification of } \\
\text { lignocellulosic biomass }\end{array}$ & 2015 & $\begin{array}{c}\text { Biochemical } \\
\text { Engineering Journal }\end{array}$ \\
\hline $\begin{array}{l}\text { Cloning and expression of l- } \\
\text { asparaginase from E. coli in } \\
\text { eukaryotic expression system }\end{array}$ & 2015 & $\begin{array}{c}\text { Biochemical } \\
\text { Engineering Journal }\end{array}$ \\
\hline
\end{tabular}

Fuente: Elaboración propia

Por último el científico Ludwig Theuvsen, departamento de Economía Agrícola y Desarrollo Rural de la Universitat Göttingen en Alemania, con 21 publicaciones, su área específica de trabajo son las cadenas de valor agroindustriales (Gollisch \& Theuvsen, 2015; Guenther-Lübbers, Bergmann \& Theuvsen, 2015; Guenther-Lübbers \& Theuvsen, 2015; Heise \& Theuvsen, 2015; Heise \& Theuvsen, 2015a; Näther \& Theuvsen, 2015; Schulte \& Theuvsen, 2015) (ver tabla 3).
Tabla 3. Producción científica reciente L. Theuvsen

\begin{tabular}{ccc}
\hline Artículo & Año & Revista \\
\hline $\begin{array}{c}\text { Animal-welfare friendly } \\
\text { control of epizootics does not } \\
\text { have to be expensive-An } \\
\text { analysis using the example of } \\
\text { classical swine fever. }\end{array}$ & 2015 & Tierarztliche Umschau \\
$\begin{array}{c}\text { Potential analysis of the biogas } \\
\text { production - as measured by } \\
\text { effects of added value and } \\
\text { employment }\end{array}$ & 2015 & Journal of Cleaner \\
\end{tabular}

$\begin{gathered}\text { Regional economic effects of } \\ \text { biogas production: An analysis } \\ \text { based on the example of Lower } \\ \text { Saxony }\end{gathered}$
$\begin{gathered}\text { Biological Functioning, Natural } \\ \text { Living or Welfare Quality: The } \\ \text { understanding of animal } \\ \text { welfare from the farmer's point } \\ \text { of view }\end{gathered}$ Landwirtschaft
$\begin{gathered}\text { German farmers' conception of } \\ \text { animal welfare: A } \\ \text { questionnaire survey }\end{gathered}$
$\begin{gathered}\text { Risk management in the farm } \\ \text { supply sector - Characteristics, } \\ \text { challenges and implications }\end{gathered}$
$\begin{gathered}\text { Influence of incentive system } \\ \text { design on individual farm } \\ \text { performance: A survey in the } \\ \text { German strawberry and } \\ \text { asparagus sectors }\end{gathered}$

Fuente: Elaboración propia

Finalmente, a nivel nacional se destaca Piedad Gañan R., de la Universidad Pontificia Bolivariana sede Medellín, con 6 publicaciones, se desempeña especialmente en la obtención de nanofibras y nanocompuestos a partir de desechos agroindustriales (Andrade et al., 2015; Álvarez et al., 2015; Castro et al., 2015; Castro, Zuluaga et al., 2015; Correa et al., 2015; Montoya et al., 2014; Osorio et al., 2014) (ver tabla 4). 
Tabla 4. Producción científica reciente P. Gañan

\begin{tabular}{|c|c|c|}
\hline Artículo & Año & Revista \\
\hline $\begin{array}{l}\text { In-situ glyoxalization } \\
\text { during biosynthesis of } \\
\text { bacterial cellulose }\end{array}$ & 2015 & Carbohydrate Polymers \\
\hline $\begin{array}{l}\text { Development of self- } \\
\text { bonded fiberboards from } \\
\text { fiber of leaf plantain: Effect } \\
\text { of water and organic } \\
\text { extractives removal }\end{array}$ & 2015 & BioResources \\
\hline $\begin{array}{l}\text { Wear resistance and friction } \\
\text { behavior of thermoset } \\
\text { matrix reinforced with } \\
\text { Musaceae fiber bundles }\end{array}$ & 2015 & Tribology International \\
\hline $\begin{array}{c}\text { Rheological and physical } \\
\text { properties of gelatin } \\
\text { suspensions containing } \\
\text { cellulose nanofibers for } \\
\text { potential coatings }\end{array}$ & 2015 & $\begin{array}{c}\text { Food Science and Technology } \\
\text { International }\end{array}$ \\
\hline $\begin{array}{l}\text { Highly percolated } \\
\text { poly(vinyl alcohol) and } \\
\text { bacterial nanocellulose } \\
\text { synthesized in situ by } \\
\text { physical-crosslinking: } \\
\text { Exploiting polymer } \\
\text { synergies for biomedical } \\
\text { nanocomposites }\end{array}$ & 2015 & RSC Advances \\
\hline $\begin{array}{l}\text { Synthesis of thermoplastic } \\
\text { starch-bacterial cellulose } \\
\text { nanocomposites via in situ } \\
\text { fermentation }\end{array}$ & 2014 & $\begin{array}{c}\text { Journal of the Brazilian } \\
\text { Chemical Society }\end{array}$ \\
\hline $\begin{array}{l}\text { Development of composite } \\
\text { films based on } \\
\text { thermoplastic starch and }\end{array}$ & 2014 & $\begin{array}{c}\text { Journal of Thermoplastic } \\
\text { Composite Materials }\end{array}$ \\
\hline
\end{tabular}

Fuente: Elaboración propia

\section{Países con mayor número de publicaciones}

Entre los países con mayor número de publicaciones relacionados, se destacan Brasil, Estados Unidos e Italia. En el caso de Brasil, las 1.169 publicaciones están relacionadas directamente con Empresa Brasileira de Pesquisa Agropecuária-Embrapa, la Universidad de Sao Paulo-USP y la Universidad Estadual PaulistaUNESP (ver figura 9).

En el caso de Estados Unidos, con 833 publicaciones, se destacan las publicaciones relacionadas con la Universidad Estatal de Michigan, la Universidad de Purdue y la Universidad de Cornell. En cuanto a la vinculación y trabajo en red para el caso de Estados Unidos se evidencia 34 publicaciones con investigadores canadienses. Finalmente, Colombia aparece en el puesto 16 con 108 publicaciones durante el periodo $2005-2015$.

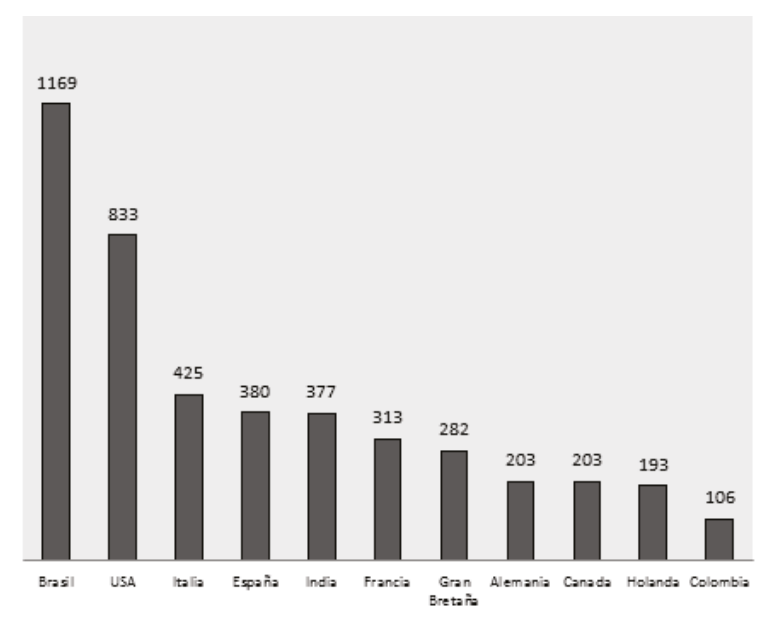

Figura 9. Países con producción científica para sector agroindustrial (2005 - 2015)

Fuente: Elaboración propia

\section{Instituciones destacadas con producción científica}

Entre las principales instituciones relacionadas con la producción científica se encuentra la Universidad de Sao Paulo, la Universidad de Wageningen y Universidad Estatal Paulista. Como se observa el $80 \%$ de las instituciones en el top 10 pertenecen a Brasil, una de Holanda y otra de Estados Unidos. A nivel nacional, en el puesto 17 con 36 publicaciones, aparece la Universidad Nacional de Colombia (ver figura 10).

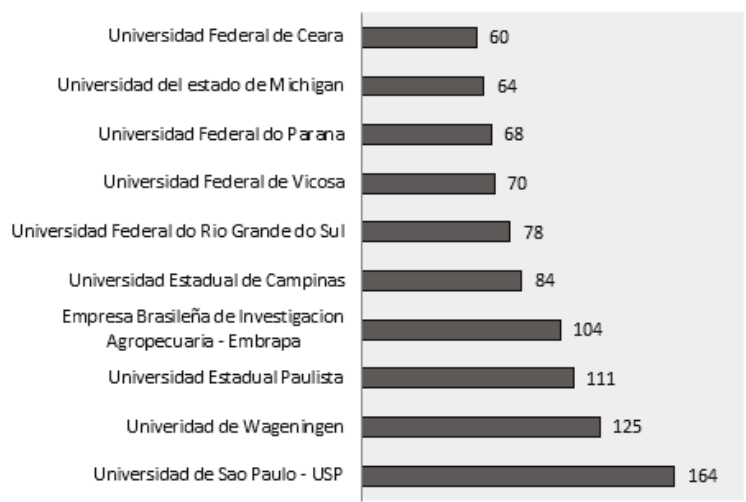

Figura 10. Instituciones con producción científica Fuente: Elaboración propia 


\section{Conclusiones}

A partir de los resultados encontrados se puede concluir que la producción científica relacionada con la agroindustria se encuentra concentrada en los países desarrollados como Estados Unidos, Italia, España, Gran Bretaña, Alemania, Canadá, entre otros; sin embargo, Brasil se constituye en el líder indiscutible, como representante de los países emergente junto con India. Este último, ubicado en quinto puesto.

Asimismo, se aprecia una tendencia altamente creciente en la producción científica en el periodo estudiado, que supera la barrera de los mil productos para 2015, con una dinámica incremental para 2016.

Frente a los campos de investigación en agroindustria, sobresales tres grandes áreas de investigación:1)Agricultura y ciencias biológicas; 2) Ciencias ambientales, y 3) Química y genética molecular. Para el área de la agricultura y ciencias biológicas, las tres principales subáreas de investigación son fermentación, seguridad alimentaria y actividad enzimática. Para el área ciencias ambientales se destacan dos subáreas de investigación: biomasa, sostenibilidad y gestión de residuos líquidos. En cuanto al área de bioquímica y genética molecular, se destacan dos subáreas de investigación; la farmacéutica y la síntesis enzimática. En general, se aprecia un alto grado de investigación de la utilidad de los subproductos y compuestos de los productos agropecuarios para el uso industrial, con alto un nivel de investigación de alta complejidad y especialización.

Con relación a los autores más productivos en las diferentes áreas de investigación, se debe resaltar de que los tres autores que más acumulan productos científicos, dos corresponden a países emergentes como Brasil e India, mientras el tercero corresponde a Alemania.

Lo anterior parece corresponder al alto potencial productor de materias primas de origen agropecuario de los dos países líderes mencionados, que buscan aceleradamente la incursión de valores agregados.

\section{Referencias Bibliográficas}

Andrade, R.D., Skurtys, O., Osorio, F., Zuluaga, R., Gañán, P. \& Castro, C. (2015). Rheological and physical properties of gelatin suspensions containing cellulose nanofibers for potential coatings. Food Science and Technology International, 21 (5), p. 332-341.

Álvarez, C., Rojas, O.J., Rojano, B. \& Ganán, P. (2015). Development of self-bonded fiberboards from fiber of leaf plantain: Effect of water and organic extractives removal. BioResources, 10 (1), p. 672-683.

Berges-García, A., Meneses-Chaus, J. M., \& Martínez-Ortega, J. F. (2016). Metodología para evaluar funciones y productos de vigilancia tecnológica e inteligencia competitiva (vt/ic) y su implementación a través de web. El Profesional de la Información, 25 (1), 103-113.

Castro, C., Cordeiro, N., Faria, M., Zuluaga, R., Putaux, J.-L., Filpponen, I., Velez, L., Rojas, O.J. \& Gañán, P. (2015). In-situ glyoxalization during biosynthesis of bacterial cellulose. Carbohydrate Polymers, 126 , p. 32-39.

Castro, C., Zuluaga, R., Rojas, O.J., Filpponen, I., Orelma, H., Londoño, M., Betancourt, S. \& Gañán, P. (2015). Highly percolated poly(vinyl alcohol) and bacterial nanocellulose synthesized in situ by physical-crosslinking: Exploiting polymer synergies for biomedical nanocomposites. RSC Advances, 5 (110), p. 90742-90749.

Cinertya Consulting. (2010). Gestión de la I+D+i con las normas de la serie UNE 166000. Barcelona.

Coral Medina, J.D., Woiciechowski, A., Zandona Filho, A., Noseda, M.D., Kaur, B.S. \& Soccol, C.R. (2015). Lignin preparation from oil palm empty fruit bunches by sequential acid/alkaline treatment - A biorefinery approach. Bioresource Technology, 194, p. 172-178. 
Correa, C.E., Betancourt, S., Vázquez, A. \& Gañan, P. (2015). Wear resistance and friction behavior of thermoset matrix reinforced with Musaceae fiber bundles. Tribology International, 87, p. 57-64.

Daim, T.U., Rueda, G., Martin, H. \& Gerdsri, P. (2006). Forecasting emerging technologies: use of bibliometrics and patent analysis. Technol. Forecasting Soc. Change 73, p. 9811012.

Da Luz Costa, J., da Silva, A.L.L., Bier, M.C.J., Brondani, G.E., Gollo, A.L., Letti, L.A.J., Erasmo, E.A.L., Soccol, C.R. (2015). Callus Growth Kinetics of Physic Nut (Jatropha curcas L.) and Content of Fatty Acids from Crude Oil Obtained In Vitro. Applied Biochemistry and Biotechnology, 176 (3), p. 892-902.

De Melo Pereira, G.V., Neto, E., Soccol, V.T., Medeiros, A.B.P., Woiciechowski, A.L. \& Soccol, C.R. (2015). Conducting starter culture-controlled fermentations of coffee beans during on-farm wet processing: Growth, metabolic analyses and sensorial effects. Food Research International, 75, p. 348-356.

Du Toit, A.S.A. (2013). Comparative study of competitive intelligence practices between two retail banks in Brazil and South Africa. J. Intell. Stud. Bus. 2, p. 30-39.

ELSEVIER. ELSEVIER. Consultado el 17 de junio de 2015 en:

http://www.americalatina.elsevier.com/ corporate/es/scopus.php.

Escorsa, P. \& Maspons, R. (2001). De la Vigilancia Tecnológica a la Inteligencia competitiva. Madrid: Prentice Hall, 320 p.

Giménez-Toledo, E. \& Román Román, A. (2001). Vigilancia tecnológica e inteligencia competitiva: conceptos, profesionales, servicios y fuentes de información. El Profesional de la Información, 10 (5), 11-20.
Gollisch, S. \& Theuvsen, L. (2015). Risk management in the farm supply sector - Characteristics, challenges and implications. Berichte uber Landwirtschaft, 93 (1).

Guenther-Lübbers, W., Bergmann, H. \& Theuvsen, L. (2015). Potential analysis of the biogas production - as measured by effects of added value and employment. Journal of Cleaner Production, 129, p. 556-564.

Guenther-Lübbers, W. \& Theuvsen, L. (2015). Regional economic effects of biogas production: An analysis based on the example of Lower Saxony. Berichte uber Landwirtschaft, 93 (2).

Heise, H. \& Theuvsen, L. (2015). Biological Functioning, Natural Living or Welfare Quality: The understanding of animal welfare from the farmer's point of view. Berichte uber Landwirtschaft, 93 (3).

Heise, H. \& Theuvsen, L. (2015a). German farmers' conception of animal welfare: A questionnaire survey. Journal of the Austrian Society of Agricultural Economics, 25, p. 117126.

Khare, S.K., Pandey, A. \& Larroche, C. (2015). Current perspectives in enzymatic saccharification of lignocellulosic biomass. Biochemical Engineering Journal, 102, p. 3844.

Lee, J. Kim, C. \& Shin, J. (2017). Technology opportunity discovery to R\&D planning: Key technological performance analysis. Technol. Forecast. Soc. Change.

Lee, S., Yoon, B. \& Park, Y. (2009). An approach to discovering new technology opportunities: keyword-based patent map approach. Technovation 29, p. 481-497.

Lee, Y., Kim, S.Y., Song, I., Park, Y. \& Shin, J. (2014). Technology opportunity identification customized to the technological capability of SMEs through two-stage patent analysis. Scientometrics 100, p. 227-244. 
Liguori, R., Soccol, C.R., de Souza Vandenberghe, L.P., Woiciechowski, A.L. \& Faraco, V. (2015). Second generation ethanol production from brewers' spent grain. Energies, 8 (4), pp. 2575-2586.

Maiti, S., Sarma, S.J., Brar, S.K., Bihan, Y.L., Drogui, P., Buelna, G., Verma, M. \& Soccol, C.R. (2015). Novel spectrophotometric method for detection and estimation of butanol in acetone-butanol-ethanol fermenter. Talanta, 141, p. 116-121.

Medina-Macedo, L., Sebbenn, A.M., Lacerda, A.E.B., Ribeiro, J.Z., Soccol, C.R. \& Bittencourt, J.V.M. (2015). High levels of genetic diversity through pollen flow of the coniferous Araucaria angustifolia: a landscape level study in Southern Brazil. Tree Genetics and Genomes, 11 (1), 814.

Montoya, U., Zuluaga, R., Castro, C., Goyanes, S., Gañán, P. (2014). Development of composite films based on thermoplastic starch and cellulose microfibrils from Colombian agroindustrial wastes. Journal of Thermoplastic Composite Materials, 27 (3), p. $413-426$.

Moreira, N.L., Dos Santos, L.F., Soccol, C.R., Suguimoto, H.H. (2015). Dynamics of ethanol production from deproteinized whey by Kluyveromyces marxianus: An analysis about buffering capacity, thermal and nitrogen tolerance. Brazilian Archives of Biology and Technology, 58 (3), p. 454-461.

Näther, M. \& Theuvsen, L. (2015). Animalwelfare friendly control of epizootics does not have to be expensive-An analysis using the example of classical swine fever. Tierarztliche Umschau, 70 (11), p. 471-477.

Osorio, M.A., Restrepo, D., Velásquez-Cock, J.A., Zuluaga, R.O., Montoya, U., Rojas, O., Gañán, P.F., Marin, D. \& Castro, C.I. (2014). Synthesis of thermoplastic starchbacterial cellulose nanocomposites via in situ fermentation. Journal of the Brazilian Chemical Society, 25 (9), p. 1607-1613.
Palop, F. y Vicente, J.M. (1999). Vigilancia Tecnológica e Inteligencia Competitiva. $\mathrm{Su}$ potencial para la empresa española. Fundación Cotec para la innovación tecnológica. Disponible en: http://www. delfos. co.cu/boletines/bsa/PDF/potencial_ vtec.pdf

Porter, M.E. (1980). Competitive Strategy: Techniques for Analyzing Industries and Competitors. New York: Free Press.

Porter, A.L. \& Cunningham, S.W. (2005). Tech mining. Exploiting New Technologies for Competitive Advantage. New Jersey: Wiley-Interscience, $180 \mathrm{p}$.

Prabisha, T.P., Sindhu, R., Binod, P., Sankar, S., Raghu, K.G. \& Pandey, A. (2015). Production and characterization of PHB from a novel isolate Comamonas sp. from a dairy effluent sample and its application in cell culture, Biochemical Engineering Journal, 101, p. 50-159.

Ramírez-Calvo, P., Triviño, A.C., Berges-García, A., Meneses-Chaux, J.M. \& Martinez, J.F.. (2013). Nuevas tecnologías en análisis de inteligencia competitiva. Casos prácticos. El profesional de la información, septiembreoctubre, v. 22 , n. 5 , p. 448-454.

Rivera, M. I. (2015). Determinant Factors for Small Business to Achieve Innovation, High Performance and Competitiveness: Organizational Learning and Leadership Style. Procedia - Social and Behavioral Sciences, 169, p. 43-52.

Sajitha, S., Vidya, J., Varsha, K., Binod, P. \& Pandey, A. (2015). Cloning and expression of 1-asparaginase from E. coli in eukaryotic expression system. Biochemical Engineering Journal, 102, p. 14-17.

Sajna, K.V., Sukumaran, R.K., Gottumukkala, L.D., Pandey, A. (2015). Crude oil biodegradation aided by biosurfactants from Pseudozyma sp. NII 08165 or its culture broth. Bioresource Technology, 191, p. 133-139. 
Sandino-Vargas, E. (2014). A strategy to meet: UK is trying to innovate in terms of competitiveness, 1998-2005. Revista Científica General José María Córdova, 12 (13), p. 205-258.

Sindhu, R., Gnansounou, E., Pandey, A. \& Binod, P. (2015). A novel crude glycerol assisted surfactant pretreatment strategy of chili post-harvest residue for bioethanol production. Biofuels, 6 (5-6), p. 383-390.

Strategic and Competitive Intelligence Professionals - SCIP. (2014). Society for Competitive Intelligence Professionals Frequently Asked Questions.

Schulte, M. \& Theuvsen, L. (2015). Influence of incentive system design on individual farm performance: A survey in the German strawberry and asparagus sectors. European Journal of Horticultural Science, 80 (5), p. 249259.

Varsha, K.K., Devendra, L., Shilpa, G., Priya, S., Pandey, A. \& Nampoothiri, K.M. (2015). 2,4-Di-tert-butyl phenol as the antifungal, antioxidant bioactive purified from a newly isolated Lactococcus sp. International Journal of Food Microbiology, 211, p. 44-50.

Villarroel, C. (2015). Diseño e implementación de una unidad de vigilancia tecnológica e inteligencia competitiva. Interciencia 40, p. 751-757.

Vivek, N., Pandey, A. \& Binod, P. (2015). Biological valorization of pure and crude glycerol into 1,3-propanediol using a novel isolate Lactobacillus brevis N1E9.3.3. Bioresource Technology, 213, p. 222-230. 